Revista Venezolana de Gerencia (RVG)

Año 16. № 54, 2011, 291 - 305

Universidad del Zulia (LUZ) • ISSN 1315-9984

\title{
Modelo de gestión de la información para los Consejos Comunales en Venezuela
}

\author{
Olivar Morillo, Cira* \\ Primera Mendoza, Nelly**
}

\section{Resumen}

El marco jurídico venezolano, otorga responsabilidades informacionales a los ciudadanos a través de mecanismos de participación, siendo uno de estos los Consejos Comunales (CC). En este sentido, el presente artículo propone un modelo de gestión de la información para los CC en Venezuela. La presente investigación es de tipo descriptiva y proyectiva, con un diseño documental y de campo, para ello se aborda teóricamente dicha gestión, mediante el análisis de los enfoques: incremento de la efectividad y ecología de la información; del mismo modo, se elaboró un diagnóstico con las limitaciones presentadas por los voceros de los CC en el cumplimiento de sus funciones informacionales.

Palabras clave: Información, Gestión de Información, Consejos Comunales.

\section{An Information Management Model for Communal Councils in Venezuela}

\section{Abstract}

The Venezuelan legal framework bestows informational responsibilities on citizens through participatory mechanisms, one of which is the Communal Council (CC). This article proposes an information management model for Communal Councils in Venezuela. The research is descriptive and projective, with a documentary, field design. It deals with said management theoretically by analyzing approaches, increasing the effectiveness and ecology of information; similarly, a diagnostic was developed with the limitations presented by $\mathrm{CC}$ spokespersons in carrying out their informational duties.

Key words: Information, information management, Communal Councils.

Recibido: 08-02-11. Aceptado: 24-05-11

* Docente de la Facultad de Ciencias Económicas y Sociales. Investigadora del Centro de Investigación y Desarrollo en Tecnologías del Conocimiento. Universidad del Zulia. Maracaibo-Venezuela. E-mail: cira_elena@yahoo.es.

** Profesora Titular Jubilada de La Universidad del Zulia. Investigadora del Centro de Investigación y Desarrollo en Tecnologías del Conocimiento. Universidad del Zulia. Maracaibo-Venezuela. E-mail: nprimera58@hotmail.com. 
Modelo de gestión de la información para los consejos comunales en Venezuela Olivar Morillo, Cira y Primera Mendoza, Nelly

\section{Introducción}

A nivel mundial, la valorización de la información en el desarrollo de los países se ubica a finales de los años setenta, cuando toma importancia en las nuevas formas de producción, en procesos organizacionales y en las relaciones sociales, fortaleciendo el denominado proyecto Sociedad de la Información, donde el procesamiento, uso y distribución de la información con el apropiado acompañamiento de las tecnologías de información y comunicación (TIC), contribuyen a la generación de conocimiento, al acceso directo e ilimitado de fuentes generadoras de información, así como a la modificación de necesidades y consumo informacional del individuo, según su nivel de educación y cultura.

Del mismo modo, aspectos de índole político y social inciden en el modo como se concibe y usa la información, evidenciado por el rol que el Estado debe cumplir como garante de la igualdad entre hombres o ciudadanos en un sistema democrático, mediante el diseño de políticas orientadas a la inclusión social en el uso de las TIC y la apropiación de la información-conocimiento a través del aprovechamiento de las mismas. En el caso venezolano, se le otorga un carácter relevante a estas dos perspectivas: a) La política-institucional, manifestada en la obligación que tienen las organizaciones de la administración pública de brindar información sobre su actuación y manejo de los distintos recursos de la organización, en términos de calidad, es decir, que sea oportuna, veraz, pertinente, vigente y precisa; y b) la social, relacionada con el derecho de acceso a la información por parte de los ciu- dadanos, la cual puede ser utilizada para acciones que contribuyan al desarrollo integral de su comunidad.

En cuanto a su aprovechamiento, desde la perspectiva institucional es útil para la mejora de los procesos organizacionales, específicamente en tomas de decisiones efectivas; y desde la social, consolida el sistema democrático participativo donde la soberanía reside intransferiblemente en el pueblo, adquiriendo el carácter de deber y derecho que tiene todo ciudadano de intervenir en la planificación, ejecución, control y evaluación de las políticas públicas que inciden en su calidad de vida, a través de diversos mecanismos de participación ciudadana entre los que se destaca para objeto de esta investigación los consejos comunales.

Estas organizaciones comunitarias en sus inicios formaban parte de la estructura y red de los Consejos Locales de Planificación Pública (CLPP) según el Art. 8 de su Ley y donde se definían los mismos como espacios de la sociedad civil, "cuya función será convertirse en el centro principal de la participación y protagonismo del pueblo en la formulación, ejecución, control y evaluación de las políticas públicas" (Asamblea Nacional, 2002); no obstante, es a partir del año 2006 con la promulgación de su marco normativo, se constituyen en instancias autónomas de participación directa, donde las actividades y competencias bajo su responsabilidad requieren además la adquisición o fortalecimiento de habilidades en materia de información.

Esta responsabilidad amerita que la información se someta a un proceso de gestión que aborde su localización, tratamiento, almacenamiento, difusión, mane- 
jo, uso, transformación o descarte de la misma cuando sea necesario, todo ello con el propósito de optimizar el levantamiento de ésta en ámbitos como el social, económico, demográfico e incluso político, así como mejorar los procesos organizacionales y generar ventajas que respondan a las necesidades prioritarias de las respectivas comunidades.

De este planteamiento, surge como objetivo del presente artículo proponer un modelo de gestión de la información (GI) para los consejos comunales en Venezuela. Para ello, se analizaron los enfoques de $\mathrm{GI}$, incremento de la efectividad (Páez, 1990) y ecología de la información (Davenport, 1999), los cuales aunado a las funciones asignadas a los consejos comunales, y a resultados obtenidos de investigaciones anteriores (Primera, Peña y Olivar, 2006; Olivar, 2008; Castrillo y Primera, 2009), constituyen la base de esta propuesta. La presente investigación es de tipo descriptiva dado que se abordaron las características esenciales de la $\mathrm{GI}$ en los consejos comunales, objeto de estudio. Del mismo modo, es una investigación de tipo proyectiva debido a que se propone un modelo de $\mathrm{Gl}$ a fin de solventar las limitaciones expuestas sobre la administración del recurso informacional en las organizaciones comunitarias, ello apoyado en lo expuesto por Hurtado (2006) que la define como aquella investigación que intenta proponer soluciones a una situación determinada a partir de un proceso previo de indagación que puede implicar la descripción del objeto de estudio.

Por otra parte, se manejo como estrategia para abordar el objeto de estudio, un diseño documental y de campo, ya que se recopilaron los principales referentes teóricos en torno a los enfoques de GI, y se elaboró un diagnóstico donde se exponen las limitaciones presentadas por los voceros de los Consejos Comunales en el cumplimiento de sus funciones informacionales, a fin de poder conocer la realidad administrativa e informacional de estas organizaciones comunitarias, aspectos que sustentan el modelo referido en este estudio.

\section{El proceso de Gestión de Información: Un abordaje teórico-conceptual}

Para el hombre la información siempre ha sido fundamental, pues constituye la base de su desarrollo y el insumo de su sistema de conocimiento que le permite intervenir en su entorno. A partir de este planteamiento, encontramos que no existen organizaciones sin información lo que podría existir son organizaciones desinformadas tal como lo plantea Ponjuán (1998), premisa ésta que se fundamenta en la idea de que todo hecho, suceso o realidad constituyen los datos que organizamos y le damos significado para convertirla en información, siempre relacionada a situaciones cotidianas de la sociedad, de las organizaciones y los individuos.

En este sentido, se puede aseverar que es de vital importancia para las sociedades actuales, tratar la información como un valioso insumo para su desarrollo siendo necesario su gestión, entendida ésta como el proceso sistémico que actúa sobre su ciclo de vida, integrado por la identificación de necesidades, generación, captura o recopilación, organización, almacenamiento, difusión, uso, 
Modelo de gestión de la información para los consejos comunales en Venezuela Olivar Morillo, Cira y Primera Mendoza, Nelly

transformación o descarte. Estas acciones son llevadas a cabo por individuos que posean habilidades y competencias informacionales.

Ahora bien, este proceso de GI presenta diversas concepciones, las cuales se especifican tomando en consideración a tres autores Páez (1990), Ponjuán (1998) y Davenport (1999), quienes la conceptualizan de la siguiente manera:

Páez (1990:63) la concibe como "el manejo de la inteligencia corporativa de una organización a objeto de incrementar sus niveles de eficacia, eficiencia y efectividad en el cumplimiento de su misión social". Destaca que el término manejo da cuenta de los procesos de planificación, conducción y evaluación de las actividades vinculadas a la gestión eficiente de la información; mientras inteligencia corporativa, se refiere a los datos, la información y el conocimiento, endógena y exógenamente generados, de valor real o potencial para asegurar la cohesión interna de la organización, su coherencia con el entorno circundante y el incremento de la productividad en las distintas funciones que contribuyen al cumplimiento de la visión organizacional.

Por su parte, Ponjuán (1998: 135) la define como "todo lo relacionado con la obtención de la información adecuada, en la forma correcta, para la persona indicada, al costo adecuado, en el tiempo oportuno, en el lugar apropiado, para tomar la acción correcta". Mientras para Davenport (1999: 169,170), representa "un conjunto estructurado de trabajo que abarcan la manera en que las compañías capturan, distribuyen y usan la información y el conocimiento".
Para fines de este trabajo, se entiende por $\mathrm{Gl}$ el conjunto de elementos y recursos que permiten a una organización la identificación de necesidades, captura, procesamiento, distribución, uso, evaluación y control de la información proveniente tanto de fuentes internas como externas, así como la generación de servicios o productos informacionales, orientados hacia el logro de objetivos, metas y fines organizacionales, y vinculadas con los procesos administrativos de planificación, organización, dirección y control.

Partiendo de estas definiciones, se puede destacar que el proceso de GI representa una herramienta gerencial que requiere de un personal capacitado para administrar este recurso intangible que incide en todos los niveles de toma de decisión, e impregna de flexibilidad y capacidad de respuesta interna y externa (disminución de incertidumbre) a la organización, generándole un valor agregado y competitivo en su mercado, consolidándola como una organización inteligente que da cuenta de un nuevo estilo de trabajo, donde la información y las TIC son recursos clave en la consecución de su misión. Desde esta perspectiva, la GI resulta un proceso complejo por la cantidad de aspectos involucrados en su aplicación. En palabras de Ponjuán (1998), exige en primer lugar, conocer perfectamente la organización (tener dominio de su filosofía de gestión, estructura, y recurso humano, entre otros) y en segundo lugar, analizar cómo se utiliza o no la información interna o externa generada o captada, por quiénes, para qué, cómo fluye o no en la misma. 
Atendiendo a estas consideraciones, se precisan a continuación dos enfoques que surgen en torno a la estructuración de la $\mathrm{GI}$, el primero basado en el incremento de la efectividad en las organizaciones, Páez (1990), y el segundo desde el enfoque de ecología de la información de Davenport (1999); ambos sirven de base para el diseño del modelo objeto de esta investigación denominada modelo sistémico de GI, la cual integra y vincula aspectos definidos en los ámbitos: organizacional-gerencial con el proceso de GI.

\subsection{Enfoque de GI: incremento de la efectividad}

Páez (1990:86-93) diseña un modelo enfocado en el incremento de la efectividad de las organizaciones, donde la estructura de este proceso se concibe desde dos perspectivas de análisis: la organización o ambiente de una GI, con los problemas que espera resolver a través de ella para satisfacer unas determinadas expectativas; y la GI propiamente, es decir, una tecnología con una capacidad determinada para utilizar un conjunto de principios y herramientas en la resolución de problemas percibidos. La operacionalización de estas dos perspectivas, requiere la ejecución de ciertas funciones, actividades y procesos, así como la disposición de cinco clases de recursos, a saber: conceptuales, humanos, de información, técnicos y tecnológicos.

Ante los niveles de dirección, procesos, actividades y decisiones más frecuentes, se hace necesario determinar necesidades de información y definir estrategias, recursos o base informacional que puedan responder a las mismas, esto sin olvidar que el proceso de GI implica la realización de auditorias informacionales. Del mismo modo, es importante el establecimiento, monitoreo y mejoramiento de los mecanismos que aseguren la cabal y económica satisfacción de necesidades señaladas o identificadas, así como la introducción y consolidación del concepto informacional en las actividades y decisiones organizacionales, esto significa garantizar una cultura informacional sana que va más allá de la capacidad para detectar y organizar la información, para extenderse hacia un flujo efectivo que alimente todos los niveles de dirección oportunamente.

Concluye Páez (1990:71) que una GI efectiva se encuentra limitada por la "ineficiencia de la gerencia, la falta de coherencia interna, la calidad de la toma de decisiones y, especialmente, la existencia de una mayor o menor resistencia al cambio" De tal modo, que su eficacia dependerá de la capacidad para apoyar la actividad gerencial y administrativa, en el desempeño de funciones y el logro de metas establecidas.

\subsection{Enfoque de GI: Ecología de la información}

Davenport (1999) presenta dos formas básicas para el estudio de la GI, una a partir de los negocios específicos y la otra del análisis de las acciones cumplidas, comprendiendo la determinación de necesidades informacionales, captura, distribución y uso, ello en correspondencia a los intereses, problemas o negocios en particular. Estas acciones, se describen a continuación: 
Modelo de gestión de la información para los consejos comunales en Venezuela Olivar Morillo, Cira y Primera Mendoza, Nelly

a) Determinación de necesidades informacionales: es una actividad ambigua y compleja, implica que ejecutivos y trabajadores entiendan el ámbito de su información, para lo cual, se requiere de la variedad de perspectivas (política, psicológica, cultural, estratégica), así como las herramientas correspondientes (evaluaciones individuales y organizacionales).

b) Captura de la información: consiste en escudriñar el entorno de la información, catalogarla en una estructura pertinente, definir su formato y presentación. Se basa en la combinación de métodos automatizados y humanos orientados a su captura interna o externa, para luego proceder a su formato (estructura) en función de las necesidades de sus usuarios. Generalmente se valen de tres fuentes: pericia externa: materiales publicados $u$ otras fuentes formales; autoridades cognitivas: individuos o instituciones que han ganado credibilidad en un campo determinado; y rumores internos de la propia organización. El desarrollo eficiente de este paso implica el manejo de dos actividades básicas:

- Catalogación: comprende la creación y definición de categorías que permitan estructurar la información, de éstas depende de manera directa la forma como se captura la misma.

- Formato y presentación: consiste en proporcionar contexto, mejorar el estilo y seleccionar el medio adecuado, para responder a las expectativas de los usuarios.

c) Distribución de la información: permite discernir cuál de los diversos medios informativos es el apropiado, y hacia donde se orienta la distribución: estrategia de oferta (se decide que información se distribuirá); de demanda (usuarios saben que requieren y que la mejor distribución es el momento de una necesidad real); o bien, la combinación de ambas en el sentido que se ofrece cierta información a los usuarios y se les permite que demanden. Se puede destacar que la distribución obedece a una variedad de grupos o intereses a los que la organización debe responder si desea lograr éxito, esto quiere decir, que "el aprendizaje organizacional ocurre no sólo a través de la captura de la información, sino a partir de distribuirla a otros" (Koening citado por Davenport, 1999:190).

d) Uso de la información: se parte de la premisa que la información no cumple su cometido hasta el momento en que se usa, por ello representa un paso clave en el proceso de GI. Se considera como algo muy personal, interno de cada individuo, puesto que en él recae el interés y la motivación de buscar, absorber y asimilar la información requerida antes de tomar una decisión, ello como consecuencia de la variedad y complejidad de problemas psicológicos y culturales que intervienen en dicha actividad. Si bien, la aplicación de este paso pudiese concebirse como algo utópico, el mismo puede llevarse a cabo en la medida que se implementen métodos pragmáticos como: mediciones (acceso, consulta a base de datos o depósitos de documentos, entre otros); actos simbólicos (recompensas, premios, declaraciones de la misión, pro- 
nunciamientos de alto nivel respecto a los valores); contextos institucionales apropiados (suministrar e intercambiar información en reuniones, juntas, consejos); y la incorporación del uso de la información en las evaluaciones del desempeño (recompensas, premios y sanciones en función no sólo de los resultados sino también de los procesos de información y decisión utilizados para tomarlas, verificando de este modo, el comportamiento y cultura informacional).

Los modelos presentados, si bien parten de la identificación de las necesidades de información para proseguir con las demás actividades ya mencionadas, no hacen énfasis en otros aspectos vinculados estrechamente con el desarrollo eficiente de una GI, pudiéndose mencionar: recursos existentes como base de datos y sistemas de información; procesos de indización, atributos de calidad y la elaboración de indicadores de gestión, por citar algunos. Desde esta lógica administrativa, algunos pueden incluirse en los pasos previstos en estos modelos, mientras que otros pudieran utilizarse para brindar amplitud al enfoque bajo el cual se concibe la GI como proceso, permitiendo de este modo precisar no sólo las actividades sino las tareas necesarias para su optimización y eficiencia en las organizaciones.

\section{Modelo de gestión de la información para los consejos comunales}

En atención a la necesidad de fortalecer la GI en los consejos comunales, se procede a esbozar los lineamientos que vinculan las funciones inmersas en el manejo de la información con los procesos administrativos que se llevan a cabo en estas organizaciones comunitarias, a través del modelo a continuación descrito.

\subsection{Modelo Conceptual}

El modelo presentado en este artículo se denomina modelo sistémico de GI, la cual tiene como objetivo definir lineamientos para administrar de manera sistémica las actividades informacionales, optimizando de este modo la satisfacción de las necesidades prioritarias de la comunidad. Ello, con la finalidad de propiciar un efectivo acercamiento de los voceros de los CC con la información y las tecnologías, que favorezca la aplicación de una buena práctica gerencial comunitaria, vinculada al proceso de la GI.

El desarrollo de este modelo es factible desde diversas perspectivas, a saber: legal: sustentando en un conjunto de leyes que promueven el acceso a la información, las tecnologías de información, y la participación ciudadana; económica: por la oportunidad que brindan los organismos nacionales e internacionales de carácter público y privado al financiamiento de proyectos de esta naturaleza; técnica: por los avances tecnológicos que permiten con facilidad la conexión a Internet y las telecomunicaciones, aunado a instituciones que brindan capacitación en el área; y social: por los beneficiarios potenciales en cada comunidad.

En este orden de ideas, es importante señalar que para diseñar este modelo se consideraron aspectos legales, teóricos y prácticos que lo sustentan. Estos fundamentos son: a) Asignación de 
Modelo de gestión de la información para los consejos comunales en Venezuela Olivar Morillo, Cira y Primera Mendoza, Nelly

responsabilidades en materia informacional a los voceros de los CC, establecidas en el marco legal que los regula. Estas son: recopilar información demográfica, social y económica de los vecinos que residen en el área de su competencia, así como elaborar un sistema de información comunitaria ${ }^{1}$ donde se recopile, organice, almacene y difunda dicha información o bien la que pudiera generarse de sus actividades diarias; b) Revisión de enfoques teóricos de GI que explican los componentes de la administración de la información como recurso gerencial; y c) un diagnostico que permitió observar las siguientes limitaciones en el cumplimiento de las asignaciones señaladas anteriormente, a saber:

- Dificultad para identificar sus necesidades de información, incorporando esta actividad a otro tipo de necesidades (básicas, sociales, como: salud, seguridad, agua, entre otras), sin interiorizar su requerimiento.

- Inexistencia de criterios en torno a la confiabilidad de las fuentes, empleando con preferencia la Alcaldía y el internet, dejando de lado a Universidades, documentos impresos, bibliotecas, por citar algunas.

- En el procesamiento de la información capturada, sólo se evidencia la selección, clasificación y almacenamiento, sin seguir criterios técnicos adecuados y uniformes. Por ejemplo almacenan en carpetas sin un méto- do de clasificación definido que facilite su posterior acceso y recuperación.

- Carencia de una base tecnológica propia, que inhibe el procesamiento electrónico de la información.

- La información recopilada de los censos o diagnóstico comunitario, pocas veces son procesadas estadísticamente para su posterior uso.

- Poca diversidad en los productos informacionales ofrecidos a la comunidad, con preferencia en hojas volantes, en pocos casos se evidenció uso de blog en internet o periódicos comunitarios.

Por otra parte, es necesario acotar que el modelo presentado profundiza los enfoques teóricos abordados, al agregar una etapa de retroalimentación como factor crucial para la mejora continúa de los procesos, incorpora el proceso de evaluación y la definición de indicadores de gestión que permitan medir o establecer la proximidad o no de la misma hacia lo planificado. Otro aporte de dicho modelo, se expresa en la vinculación directa que se establece entre los procesos administrativos y las funciones de la GI, basado en la conexión que existen entre estos, tal como se señala en el Gráfico 1.

En este modelo, se parte de la noción que un proceso de GI por su transversalidad debe tomar en consideración los procesos administrativos, entrelazando las funciones de GI con la realidad ad-

1 Con la modificación de su ley, recién aprobada en Noviembre de 2009, el Sistema de Información Comunitaria, es responsabilidad exclusiva de la Unidad Ejecutiva de los consejos comunales, de acuerdo a lo establecido en el artículo 29, numeral 2. 


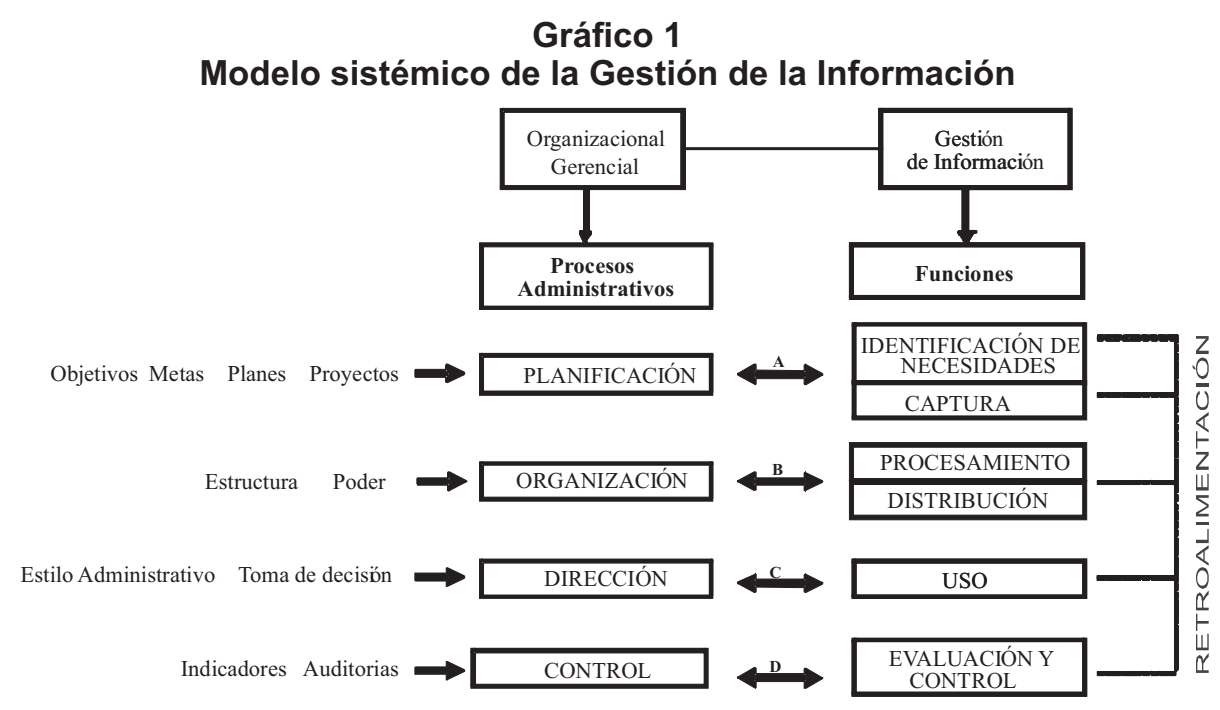

Fuente: Elaboración Propia.

ministrativa de los CC, de manera que respondan a la razón de ser de los mismos, delimitando el accionar y aprovechamiento de la información.

En tal sentido, se definieron dos ámbitos de análisis:

- Organizacional-Gerencial: integrado por la planificación (expresados a través de objetivos establecidos, planes, proyectos), organización (estructura organizacional, concentración del poder), dirección (estilo administrativo, toma de decisiones) y control (indicadores de gestión). Cada uno de estos procesos direccionan la información requerida en la medida en que se cumplan.

- Gestión de Información: referido a las funciones necesarias para gestionar este recurso, a saber: identificación de necesidades informacionales, captura, procesamiento, distribución, uso y evaluación-control de la información requerida, esta última en función de retroalimentar el proceso con el fin de la mejora continua, detectar posibles desviaciones y aplicar medidas correctivas.

Para un mejor conocimiento del modelo, a continuación se explica la vinculación establecida:

a) Planificación con identificación de necesidades información y, captura: dada por la necesidad que tienen los $\mathrm{CC}$, de desarrollar planes y proyectos orientados a acciones precisas que permitan el logro de objetivos y metas formuladas. A partir de estos lineamientos surgen determinadas necesidades de información que de acuerdo a la naturaleza del área organizacional permitirá establecer el tipo requerido, sea ésta económica, financiera, política, cultural, sobre merca- 
Modelo de gestión de la información para los consejos comunales en Venezuela Olivar Morillo, Cira y Primera Mendoza, Nelly

deo, entre otras, así como las fuentes donde localizarla.

b) Organización con procesamiento $y$ distribución: este proceso incide en el modo como será procesada, presentada y distribuida la información, ya que ilustra jerarquías, división de funciones, roles y la agrupación de actividades que dentro de ella pueden darse, aunado a las posibles relaciones de poder y autoridad que se tejen en su dinámica (formal e informal), dada por las expectativas e intereses mutuos de sus miembros, la interrelación y sus comportamientos, e incluso, la cultura organizacional e informacional, es decir, la voluntad y compromiso por compartir la información generada, capturada y procesada. Una estructura organizacional diseñada de manera correcta, incide favorablemente en el uso de sistemas de información y comunicación.

La estructura organizacional de los $\mathrm{CC}$, en su reciente reforma (Gaceta Oficial N $N^{\circ} 39.335$ de 28 de Diciembre de 2009), establece que éstos para fines de su funcionamiento estarán integrados por: la Asamblea de Ciudadanos, el Colectivo de Coordinación Comunitaria y las unidades Ejecutiva, Administrativa y Financiera Comunitaria, y de Contraloría Social, éstas últimas integradas por los voceros electos (principales) con sus respectivos suplentes, aspecto innovador en la organización de los $\mathrm{CC}$, en conjunto con el sistema de coordinación colectiva antes no previsto (Ver Gráfico 2). Esta estructura deja a un lado la ordenación tradicional jerárquica-ver- tical-lineal, para responder a una horizontalidad, basada en principios de trabajo como la cooperación, rendición de cuentas, eficacia, eficiencia, trabajo voluntario, por mencionar algunos. Sus comités de trabajo y unidades funcionan de manera articulada, con el mismo grado de participación, responsabilidad y atribuciones en concordancia a cada área contemplada en el mismo, siendo éstas cambiantes de acuerdo a la naturaleza y necesidades de la comunidad en la que se encuentre localizado.

c) Dirección con uso de la información: representa el proceso administrativo a través del cual se aplican los conocimientos (información ya analizada) en la toma decisiones, y se direcciona, motiva e impulsa al recurso humano a lograr los objetivos organizacionales, donde influye la capacidad de liderazgo sobre el comportamiento de la gente como miembro de un grupo. La calidad de este proceso se produce cuando se usa información de calidad para tomar decisiones, resolver problemas, formular planes, programas y proyectos, alcanzar objetivos, implementar métodos de organización, trabajo, lograr formas de conducta más deseables, y sistemas de control, por mencionar algunos.

d) Control con evaluación: a través del cual se evalúa el rendimiento, llevando una adecuada supervisión de las actividades realizadas en función de lo previamente planificado y la definición de estándares o patrones para determinar posibles desviaciones, todos los involucrados deben participar en el mismo, ya que permite la retroa- 


\section{Gráfico 2}

\section{Estructura de los Consejos Comunales}

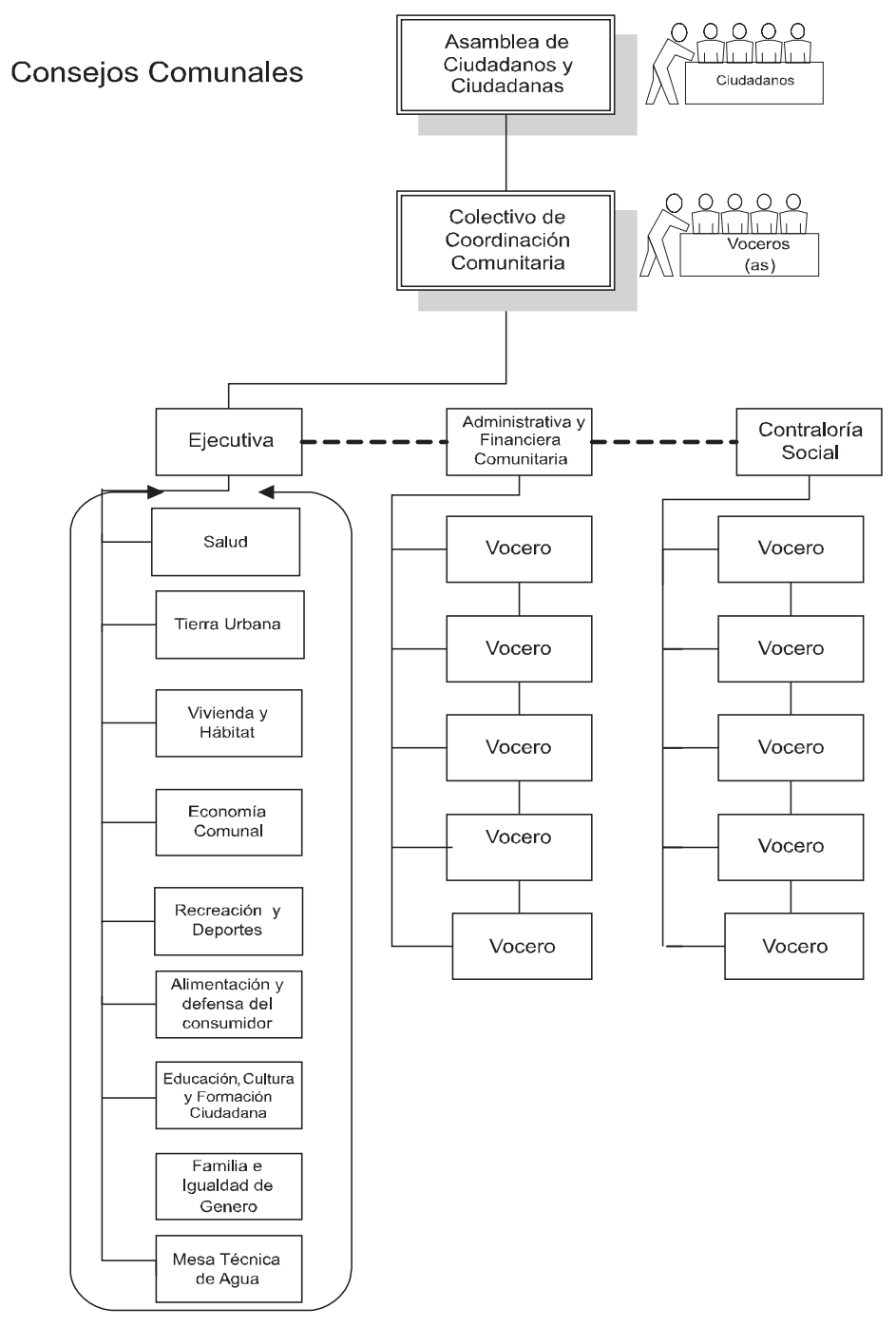

Leyenda:

Coordinación y cohesión entre las unidades - - - - -

Comités de trabajo por área clave de las comunidades

Fuente: Elaboración propia con información tomada de la Ley de los Consejos Comunales (2009). 
Modelo de gestión de la información para los consejos comunales en Venezuela Olivar Morillo, Cira y Primera Mendoza, Nelly

limentación de información que sustentará a la planificación, haciendo que la misma se ajuste a la realidad organizacional, ello viéndolo como un proceso continuo de supervisión y evaluación (control concomitante), aplicado durante todo el proceso y no sólo en los resultados finales o en la planificación.

Queda así plasmada, la vinculación del modelo sistémico de GI para los CC en Venezuela, establecida por la realidad administrativa y las funciones de GI que en los mismos puede implementarse para un funcionamiento exitoso.

\subsection{Aplicabilidad del Modelo}

El cúmulo de información que llega a los CC producto de la globalización y el avance vertiginoso de las tecnologías, ocasiona problemas relacionados con la saturación, subutilización y la desinformación, debido a la ausencia de categorías para su selección, análisis, almacenamiento y distribución efectiva tanto interna como externa, esto evidencia la necesidad de cumplir un proceso de GI -previsto como el modelo propuesto- en estas organizaciones comunitarias, con el fin de mejorar sus procesos tanto organizativos como informacionales. Para la adaptación del modelo propuesto, se definen las siguientes fases:

Primera Fase. Planificación.

Comprende las acciones previas de formación del talento humano, apoyo institucional y fuentes de financiamiento.

a) Formación del Talento humano:

- Definir programas educativos que contemplen las herramientas míni- mas para administrar la información, en áreas como: identificación de necesidades informacionales, selección de fuentes, procesamiento, entre otros.

- Desarrollar competencias informacionales en los voceros de los CC y prepararlos como facilitadores. Empleando técnicas como talleres, conversatorios, lecturas dirigidas, por citar algunas.

- Establecer acuerdos con Instituciones de Educación Superior (preferiblemente con Escuelas de Bibliotecología y Archivología, Informática, Comunicación Social, Administración y Sociología), para que a través de la aplicación del Servicio Comunitario del Estudiante de Educación Superior contemplado en el país, se desarrollen y apliquen los módulos para la Alfabetización Informacional de los voceros de los CC, quiénes una vez formados, actuarán como facilitadores para el resto de la comunidad.

- Concienciar a la comunidad sobre el valor de la información y la necesidad de gestionarla como recurso esencial para el funcionamiento de los CC.

b) Apoyo Institucional y fuentes de financiamiento:

- Difundir las bondades del modelo a las autoridades del Ministerio del Poder Popular para las Comunas, gobiernos regionales y municipales.

- Incorporar instituciones públicas y privadas como fuentes de financiamiento del proyecto, que incluya dotación y mantenimiento de equipos, así como asesoría o acompañamiento técnico. 

vidad.

Segunda Fase: Procesos y operati-

Según lo planteado en el modelo es necesario llevar a cabo las siguientes acciones:

- Realizar un diagnóstico informacional a cada CC, con el fin de determinar las acciones informacionales cumplidas y las posibles limitantes.

- Cotejar el modelo de GI propuesto con la situación detectada en cada CC, para mejorar los procesos, mediante la aplicación de medidas correctivas.

- Estandarizar los procesos administrativos para facilitar la GI.

- Crear el Sistema de Información Comunitario (SIC) como herramienta de apoyo a la GI, a través de funciones tales como: captura y procesamiento de la información, transferencia de documentos, generación de productos, entre otras.

- Diseñar controles (concomitantes preferiblemente) que permitan evaluar la efectividad del modelo.

Tercera Fase: Indicadores de logro.

Los indicadores de logro contemplados para determinar el grado de avance en la aplicabilidad del modelo propuesto, son los siguientes:

- Incremento del número de voceros y ciudadanos alfabetizados informacionalmente.

- Apoyo de los voceros para la aplicación del modelo de GI.

- Incorporación de tecnologías de información para la recuperación, procesamiento y distribución de la información.
- Efectividad en el proceso de toma de decisiones.

- Facilidad de acceso a información de calidad.

- Índice de uso de los SIC.

- Estandarización de los procesos administrativos.

- Satisfacción de las necesidades de información de las comunidades y de los ciudadanos.

- Cambios en la cultura informacional en los sectores, parroquias y municipio.

- Número de SIC instalados en el municipio.

\section{Conclusiones}

Considerando que la GI es una herramienta clave para el manejo eficiente de un recurso tan especial, que actúa como eje transversal en todos y cada uno de los procesos, sistemas y servicios que se ejecutan en las organizaciones, es necesario establecer su estrecha vinculación con la realidad administrativa. En el caso de los CC, dicha gestión constituye el medio a través de la cual se puede responder eficazmente a la responsabilidad de levantamiento de información social, económica, cultural, demográfica e incluso política, asignadas en el marco legal que regula estos mecanismos de participación ciudadana.

Surge la propuesta presentada como una alternativa para la optimización y eficiencia de la información en estas organizaciones comunitarias, propiciando en sus actores una cultura que valorice a ésta como insumo gerencial clave para el 
Modelo de gestión de la información para los consejos comunales en Venezuela Olivar Morillo, Cira y Primera Mendoza, Nelly

cumplimiento de sus respectivas responsabilidades. La ausencia de esta cultura pudiera moldear, en los voceros comunitarios, un comportamiento ajeno a velar por los intereses del colectivo, al ejercicio del control social, a la participación crítica y efectiva en la formulación de las políticas públicas que les atañen a las comunidades, entre otras.

La presencia de problemas informacionales en los $\mathrm{CC}$, trae consigo actitudes poco favorables para la toma de decisiones acertadas y acciones eficientes que atiendan demandas y satisfagan necesidades sentidas por las comunidades, dando paso a la saturación de información irrelevante, desactualizada, sin veracidad, imprecisa; acumulando en cierto modo, datos que no se traducen en información útil por la falta de sistematicidad de la misma. Revertir estas ausencias, implica plantearse estrategias muy puntuales en alfabetización informacional, la cual pasa por elaborar y ejecutar programas educativos que desarrollen en los sujetos comunitarios aquellas competencias básicas para el acceso, procesamiento y comunicación de la información, que propicien el desarrollo y sostenibilidad de sistemas de información comunitaria.

Para ello, debe haber un sentido de responsabilidad y acompañamiento por parte de los entes públicos competentes en las áreas: gerencial e info-tecnológicas, la primera comprende la definición clara de aspectos organizativos y gerenciales propios de los consejos comunales, siendo evidente por ejemplo, la necesidad de concienciar a sus integrantes en que el objeto de la contraloría social no sólo son las empresas o sujetos externos sino que se debe supervisar y evaluar la gestión interna, basado en principios como la eficacia, eficiencia, productividad, calidad y bienestar social.

Mientras la segunda se traduce en planificar y ejecutar programas para el desarrollo de competencias que propicien en los ciudadanos un mejor uso de la información. Ambas acciones permitirían el funcionamiento de estructuras info-tecnológicas (tecnología, sistemas de información automatizados, conformación de redes comunitarias, acceso a Internet, entre otros) con la finalidad de mejorar su imagen institucional y posicionarse en sus comunidades y en la sociedad venezolana.

En síntesis, se puede aseverar que la GI en este tipo de organización (instancia de participación ciudadana) representa un proceso complejo que amerita de voluntad política-institucional y de organización de las propias comunidades, representando esto último un reto, en el entendido de afianzar en los líderes comunitarios el compromiso por el bienestar social, canalizado no por la improvisación sino por el manejo de herramientas gerenciales, informacionales y organizativas que les de garantía de una gestión exitosa. Sólo el tiempo, el trabajo día a día, la voluntad política de los dirigentes nacionales, regionales y principalmente locales, y la vocación de servicio de los funcionarios públicos, determinarán la rapidez o no de los avances en esta materia. 


\section{Referencias bibliográficas}

Asamblea Nacional (2009). Ley de los Consejos Comunales. Gaceta Oficial $N^{\circ}$ 39.335. Caracas, Venezuela.

Asamblea Nacional (2006). Ley de los Consejos Comunales. Gaceta Oficial Extraordinaria $\mathrm{N}^{\circ}$ 5.806. Caracas, Venezuela.

Asamblea Nacional (2002). Ley de los Consejos Locales de Planificación Pública. Gaceta Oficial № 37.463. Caracas, Venezuela.

Castrillo, Dayana y Primera, Nelly (2009). Procesos medulares en el desarrollo de competencias informacionales en los consejos comunales del municipio Mara: Parroquia Ricauter. Trabajo Especial de Grado. Escuela de Bibliotecología y Archivología de la Facultad de Humanidades y Educación. Universidad del Zulia. Maracaibo, Venezuela.

Davenport, Thomas (1999). Ecología de la Información. Oxford University Press. México.

Hurtado, Jacqueline (2006). El proyecto de investigación. Metodología de la In- vestigación Holística. 4ta. Edición. Ediciones Quiron. Bogotá, Colombia.

Páez, Iraset (1990). Información para el progreso de América Latina. Universidad Simón Bolívar. Caracas, Venezuela.

Ponjuán, Gloria (1998). Gestión de Información en las organizaciones. Principios, conceptos y aplicaciones. Universidad de Chile, Centro de Capacitación en información. Santiago de Chile.

Primera, Nelly; Peña, Dionnys y Olivar, Cira (2006). Portafolio de Productos y servicios basados en tecnologías de información para la consolidación de los Consejos Locales de Planificación Pública (CLPP) en el estado Zulia. Informe Final. Ministerio de Ciencia y Tecnología. Fonacit. Maracaibo, Venezuela.

Olivar, Cira (2008). Gestión de Información en los Consejos Comunales del municipio Maracaibo del estado Zulia (2006-2007). Trabajo Especial de Grado. División de Estudios para Graduados de la Facultad de Ciencias Económicas y Sociales. Universidad del Zulia. Maracaibo, Venezuela. 